\title{
Influence of backside metallization on a coplanar X-band LNA
}

\author{
R. Follmann, G. Langgartner, J. Borkes and I. Wolff \\ IMST GmbH, D-47475 Kamp-Lintfort, Germany, e-mail follmann@imst.de \\ H.-P. Feldle \\ EADS Deutschland GmbH, D-89070 Ulm, Germany
}

\begin{abstract}
In this paper the influence of the backside metallization on a coplanar X-band low noise amplifier MMIC is described. First, we demonstrate the selection of transistor devices including the modelling especially with respect to the extraction of RF noise parameters. After that the simulation results and the layout of the LNA are presented, neglecting backside effects on coplanar circuits. Completing measurements on the fabricated LNA show the influence of the backside metallization in comparison with simulation results.
\end{abstract}

\section{SELECTING AND MODELling OF THE TRANSISTOR DEVICES}

Transistor devices used in LNAs must fulfill one important criterion: Using a corresponding circuitry, the transistor device must have both a good noise match as well as a good power match. Keeping this in mind, we looked for transistor devices that are suitable for the LNA specifications $f=8 . .12 \mathrm{GHz}$, gain $=18 \mathrm{~dB}$, $N_{\mathrm{f}} \leq 1.4 \mathrm{~dB}, P_{1 \mathrm{~dB}}=12.0 \mathrm{dBm}$ and TOI $\geq 22.0 \mathrm{dBm}$. For noise parameter extraction and simulation, we used the TOPAS equivalent circuit [1] as shown in fig. 1. The ten nodes of the equivalent

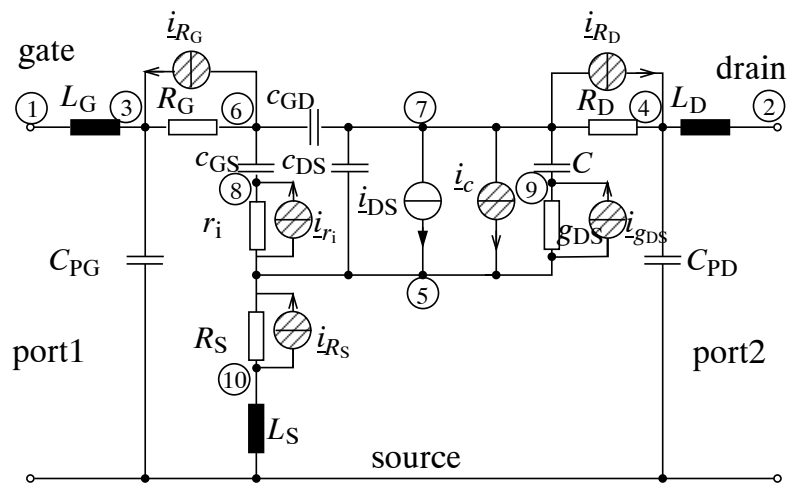

Fig. 1. Noisy equivalent circuit for a FET.

circuit are numbered in order to determine the $10 \times 10 \mathrm{Y}$-matrix. In correspondence with the Y-matrix, a $10 \times 10$ noise matrix can be defined. The position of noise sources in that matrix is given by the node numbers at the end of the noise source. The direction of the current is defined in such a way that at matrix entry $i_{\mathrm{j}, \mathrm{k}}$ the noise current in the equivalent circuits flows from node $\mathrm{j}$ into the direction of node $\mathrm{k}$. Thus, all noise current sources have only positive entries. The common factor $\sqrt{4 k T \Delta f}$ is placed outside the brackets. For the presented equivalent circuit, the noise current matrix is given by

$$
\begin{gathered}
{\left[i_{\mathrm{N}}\right]=\sqrt{4 k T \Delta f} .} \\
\left(\begin{array}{cccccccccc}
0 & 0 & 0 & 0 & 0 & 0 & 0 & 0 & 0 & 0 \\
0 & 0 & 0 & 0 & 0 & 0 & 0 & 0 & 0 & 0 \\
0 & 0 & 0 & 0 & 0 & 0 & 0 & 0 & 0 & 0 \\
0 & 0 & 0 & 0 & 0 & 0 & 0 & 0 & 0 & 0 \\
0 & 0 & 0 & 0 & 0 & 0 & 0 & i_{5,8} & i_{5,9} & 0 \\
0 & 0 & i_{6,3} & 0 & 0 & 0 & 0 & 0 & 0 & 0 \\
0 & 0 & 0 & i_{7,4} & i_{7,5} & 0 & 0 & 0 & 0 & 0 \\
0 & 0 & 0 & 0 & 0 & 0 & 0 & 0 & 0 & 0 \\
0 & 0 & 0 & 0 & 0 & 0 & 0 & 0 & 0 & 0 \\
0 & 0 & 0 & 0 & i_{10,5} & 0 & 0 & 0 & 0 & 0
\end{array}\right)
\end{gathered}
$$

with the resistive noise

$$
\begin{aligned}
i_{5,8} & =\sqrt{\frac{1}{r_{\mathrm{i}}}}, \\
i_{5,9} & =\sqrt{g_{\mathrm{DS}}}, \\
i_{6,3} & =\sqrt{\frac{1}{R_{\mathrm{G}}}}, \\
i_{7,4} & =\sqrt{\frac{1}{R_{\mathrm{D}}}}, \\
i_{7,5} & =\sqrt{g_{\mathrm{i}_{\mathrm{c}}}}, \\
i_{10,5} & =\sqrt{\frac{1}{R_{\mathrm{S}}}}
\end{aligned}
$$

including the channel noise

$$
g_{\mathrm{i}_{\mathrm{c}}}=\frac{T_{\mathrm{sim}}}{T_{0}} \frac{2}{3} g_{\mathrm{m}}+k_{f} \frac{I_{c}^{a f}}{f^{b} 4 k T_{0}} .
$$

As shown in [2], the noise figure can be calculated using the equations above to

$$
F=1+\frac{T_{\text {sim }}}{T_{0}} \frac{\left|\underline{y}_{\mathrm{G}}\right|^{2} C_{11}^{(a)}+C_{22}^{(a)}+2 \Re\left\{\underline{y}_{\mathrm{G}} C_{12}^{(a)}\right\}}{g_{\mathrm{G}}} .
$$

For the extraction of noise parameters, this calculated noise figure can be compared to the measured one to achieve the required RF noise parameters of the transistor device.

\section{THE LNA DESIGN}

For the design of the LNA, the UMS T624 transistor was chosen. This transistor is a $6 \times 40 \mu \mathrm{m}$ HEMT device delivering excellent 
noise performance and satisfying gain. Fig. 2 shows the principal set up of the LNA. The whole LNA consists of two stages.

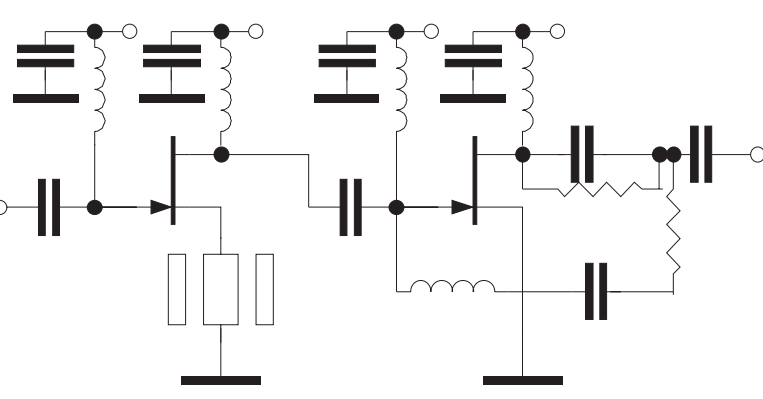

Fig. 2. Principal circuit of the LNA.

The first stage features a serial inductive feedback. This causes a falling gain over frequency, which is compensated by the second stage. Therefore, this stage features a parallel inductive feedback. Both stages are matched at input and output using shorted inductors. DC input is connected over these inductors, too. For the decoupling of DC, capacitors are used at the input and output port as well as between the stages. The parallel circuitry consisting of a capacitor and a resistor at the output of the second stage takes care of a constant efficiency and delivers more stability. One critical element in the layout of the LNA is the parallel circuit consisting of a MIM capacitor and a resistor at the output of the second transistor stage. Due to the fact that this element is not contained in the coplanar library we used, it has been simulated using a MIM capacitor in parallel with an ideal resistor. This should result in a sufficient grade of accuracy for X-band frequencies. The LNA was produced at UMS with the $\mathrm{PH} 25$ process on a thinned wafer $(100 \mu \mathrm{m})$ with backside metallization. This metal is needed for mounting and stability reasons.

\section{Simulation RESUlts}

The LNA operates at the bias conditions $U_{\mathrm{G} 1}=U_{\mathrm{G} 2}=-0.3 \mathrm{~V}$ and $U_{\mathrm{D} 1}=U_{\mathrm{D} 2}=3.0 \mathrm{~V}$. All simulations have been carried out at a temperature of $27{ }^{\circ} \mathrm{C}$. The current consumption is $73 \mathrm{~mA}$. The simulation of the scattering parameters $s_{11}, s_{21}$ and $s_{22}$ can be seen in the next fig. (3). Input and output matching is better than 14

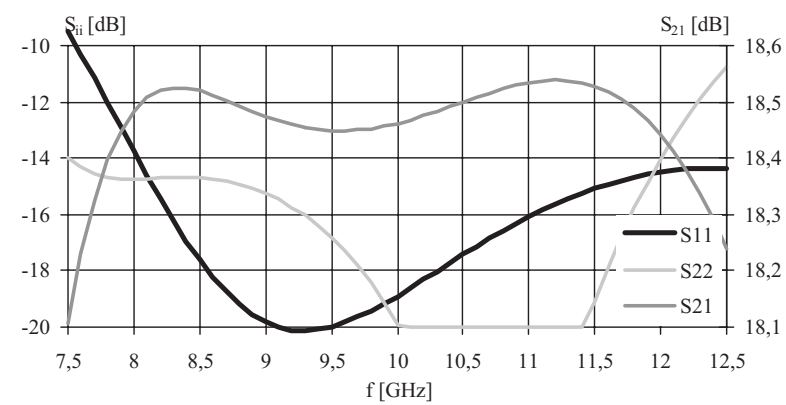

Fig. 3. Simulated scattering parameters of the LNA.
$\mathrm{dB}$, the gain $18.5 \mathrm{~dB}$. As the k factors (fig. 4) demonstrate, the second stage may become instable. Nevertheless, a closer look at the stability circuits shows that all circuits of instability are far away from the input and output impedances of the LNA. Thus, the

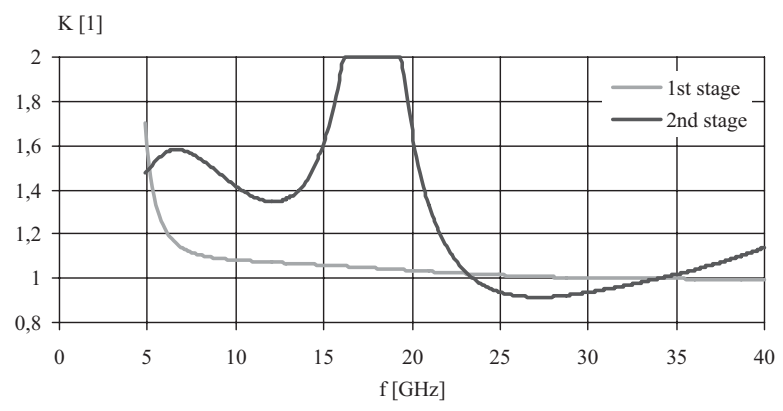

Fig. 4. K factors of the LNA.

LNA is conditionally unstable, but stable in the frequency range of interest. The final layout of the LNA can be seen in fig. 5. The

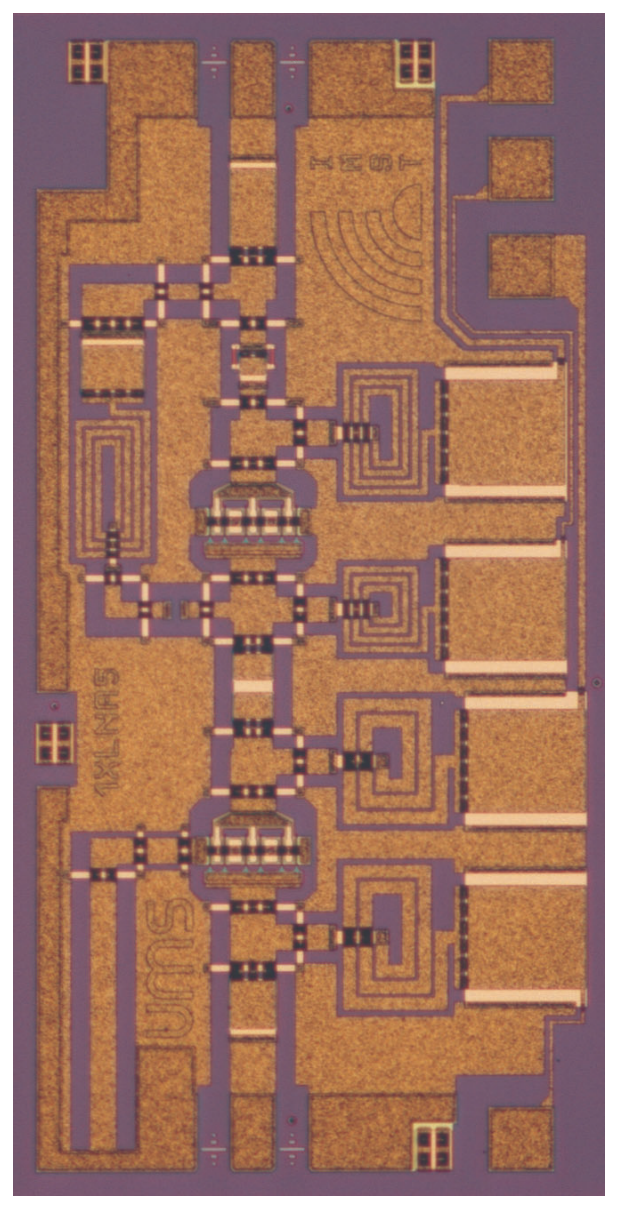

Fig. 5. Final layout of the LNA.

MMIC size is $1.80 \times 0.89 \mathrm{~mm}^{2}\left(1.60 \mathrm{~mm}^{2}\right)$. The simulation of the noise figure and the minimum noise figure (fig. 6) demonstrates 
that the noise matching was done optimally.

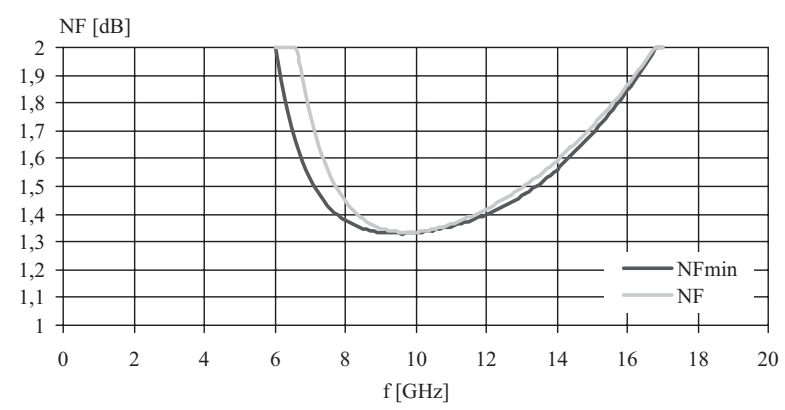

Fig. 6. Noise figure and minimum noise figure of the LNA.

\section{Measurements}

To exclude external influence and disturbance, the MMIC was mounted on a ceramic. All DC bond pads were connected to bypass capacitors $(1000 \mathrm{pF}$ and $47 \mu \mathrm{F})$. The gate voltage was

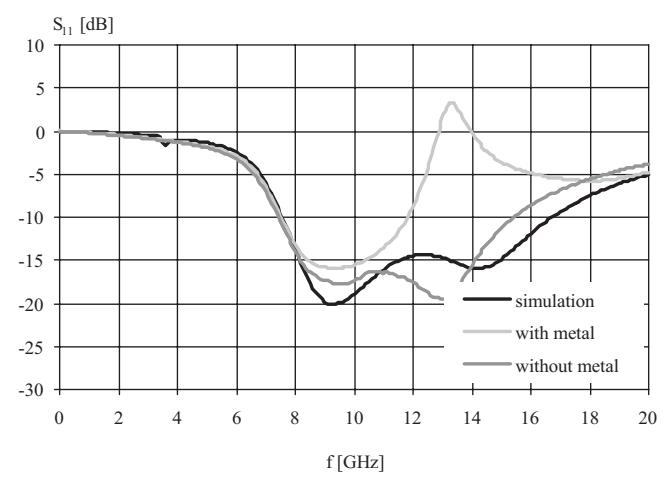

Fig. 7. Scattering parameters of the LNA. Simulation versus measurement for $s_{11}$.

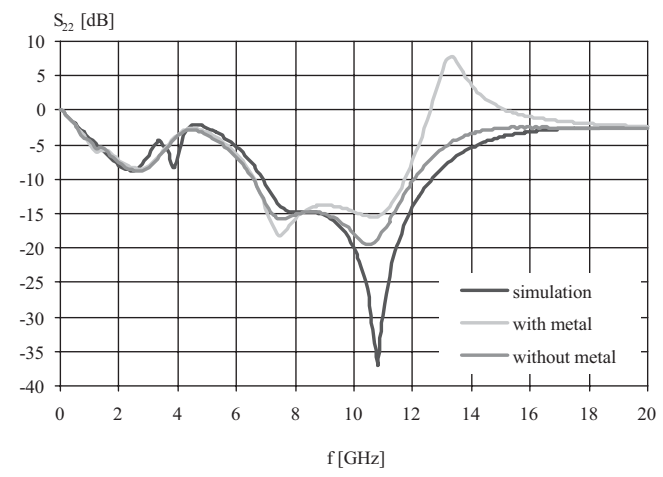

Fig. 8. Scattering parameters of the LNA. Simulation versus measurement for $s_{22}$. connected through a $500 \Omega$ resistor. Despite these precautions the measured scattering parameters showed resonances at approximately $13 \mathrm{GHz}$. Further examinations with a spectrum analyzer result in the fact, that no oscillations could be observed, although the resonance effects in the scattering parameters led to this suspect. It turned out more and more, that the resonant behavior was caused by substrate modes due to the backside metallization of the MMIC as described in [3] for mounting on different surfaces. Therefore, the backside metal of the MMIC was removed using a gold etchant and hydrofluoric acid. To

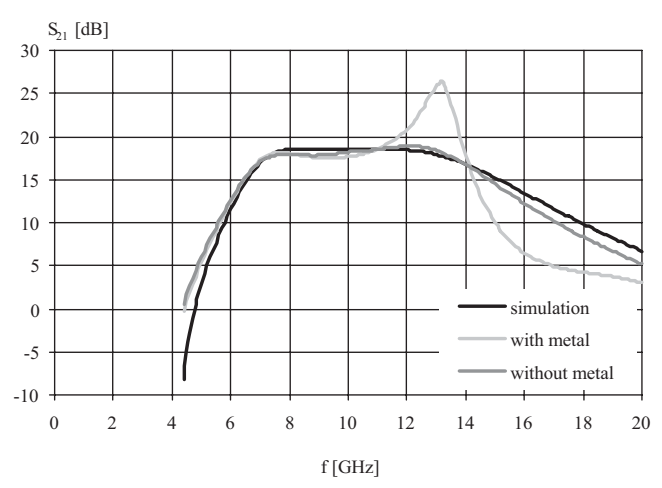

Fig. 9. Scattering parameters of the LNA. Simulation versus measurement for $s_{21}$.

protect the MMIC front against this etch solution, it was dipped into wax before the etching process started. After some trials we managed to remove the backside metallization successfully. After this treatment new measurements have been carried out with great success. All resonance effects disappeared and the measurement agrees very well with the simulation. The four figures (fig. 7-10) show the simulation of the LNA scattering parameters versus two measurements. The first measurement included the backside

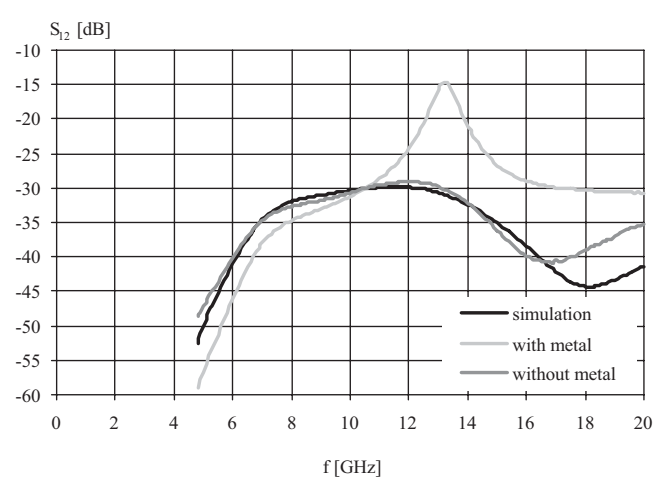

Fig. 10. Scattering parameters of the LNA. Simulation versus measurement for $s_{12}$.

metal, the second not.

The measurements demonstrate the influence of the backside metallization. After removing it, they agree perfectly with the simulations. Also the measurement of the noise figure agrees 


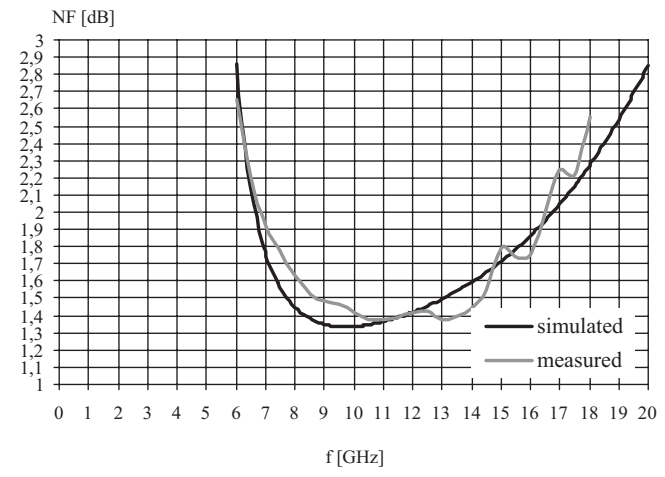

Fig. 11. Simulated versus measured noise figure of the LNA.

12 the simulated versus the measured output power can be seen. After removing the backsite metal both results agree very well.

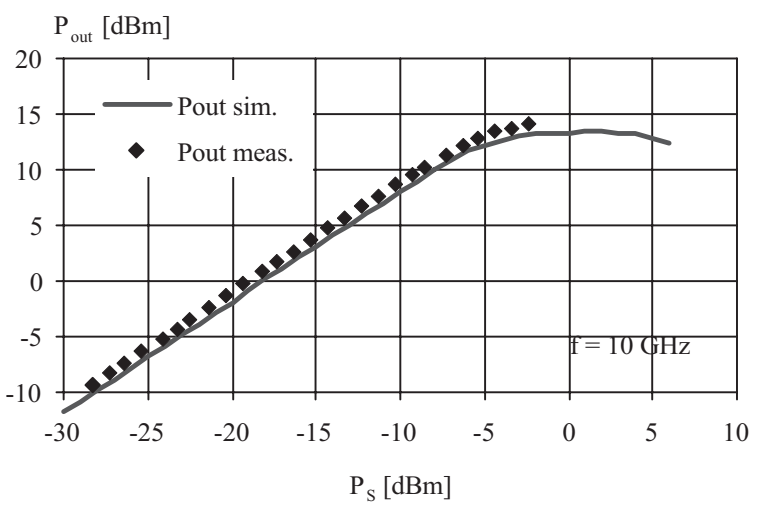

Fig. 12. Simulated and measured output power of the LNA.
Due to heat sinking capabilities, stability, and mounting reasons, coplanar MMICs are often fabricated with a backside metal on a thinned $(100 \mu \mathrm{m})$ wafer. We demonstrated on a well designed low noise amplifier how the backside metal affects the performance of the whole MMIC. With backside metal, parallel plate modes are stimulated and the LNA shows resonances in the scattering parameters, although it does not oscillate. Without the metal the measurement results agree perfectly with the simulation. The possibility of stimulating a parallel plate mode is nearly independent of the frequency because these modes propagate as from $f=0 \mathrm{~Hz}$. We obtained similar effects on ka-band circuits. A thicker wafer attenuates the influence of the parallel plate mode due to higher resistivity. Today, these effects on coplanar circuits cannot be simulated with state-of-the-art design software.

\section{REFERENCES}

[1] R. Follmann, J. Borkes, P. Waldow, and I. Wolff, "Extraction and Modeling Methods for FET devices," IEEE Microwave Magazine, vol. 1, no. 3, pp. 49$55,2000$.

[2] R. Follmann, J. Berben, D. Köther, and I. Wolff, “A universal Method for Calculating and Extracting the LF and RF noise Behaviour of Nonlinear Devices," 22nd IEEE GaAs IC Symposium, Seattle, Washington, vol. 1, pp. 47$50,2000$.

[3] T. Krems, A. Tessmann, W. H. Hyadl, C. Schmelz, and P. Heide, "Avoiding cross talk feedback effects in packaging coplanar millimeter-wave circuits," IEEE Trans. Microwave Theory Tech., pp. 1-4, 1998. 